\title{
Varón de 57 años con episodios recurrentes de rinorrea acuosa desencadenados por cambios bruscos de posición
}

\author{
Herrero Sanz Ma J. ${ }^{1}$, Hernández Sánchez G. ${ }^{2}$
}

Sanid. mil. 2014; 70 (1): 49-50; ISSN: 1887-8571

Varón de 57 años que acude al servicio de ORL por presentar desde hace un año episodios de rinorrea acuosa con descarga brusca de líquido ambarino y claro desencadenados por cambios de posición cefálicos. Antecedentes personales: HTA mal controlada. Exploración ORL: rinoscopia y resto de la exploración aparentemente normal. Exploraciones complementarias: ante la sospecha de fístula de LCR se realizó una Tomografía Computerizada (TC) de senos paranasales en la que se apreció la ocupación del seno esfenoidal derecho por un nivel hidroaéreo (fig.1) y una imagen sugerente de solución de continuidad en la pared externa del mismo; se recomendó la valoración por otros medios diagnósticos. El estudio se completó con una cisternografía mediante Tomografía Computerizada Multicorte (TCMC).

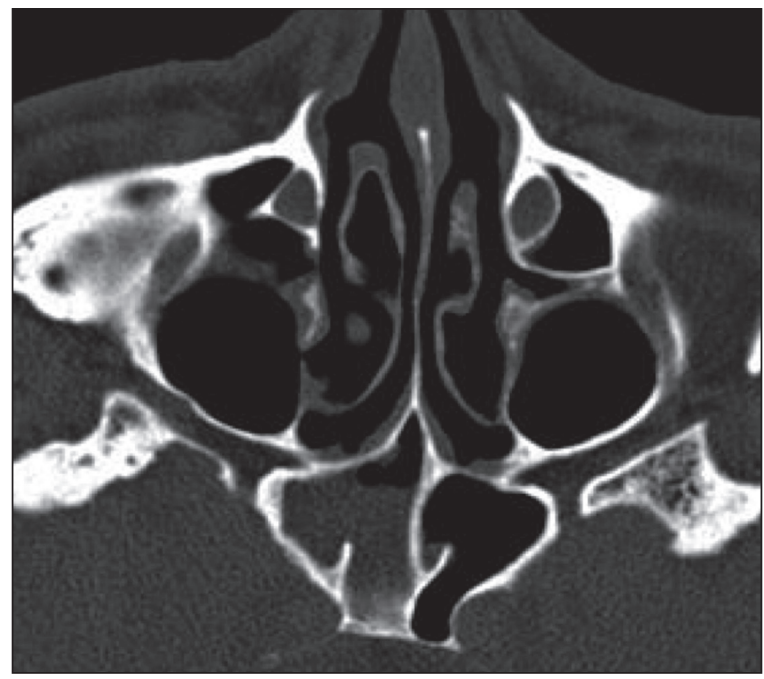

Figura1.

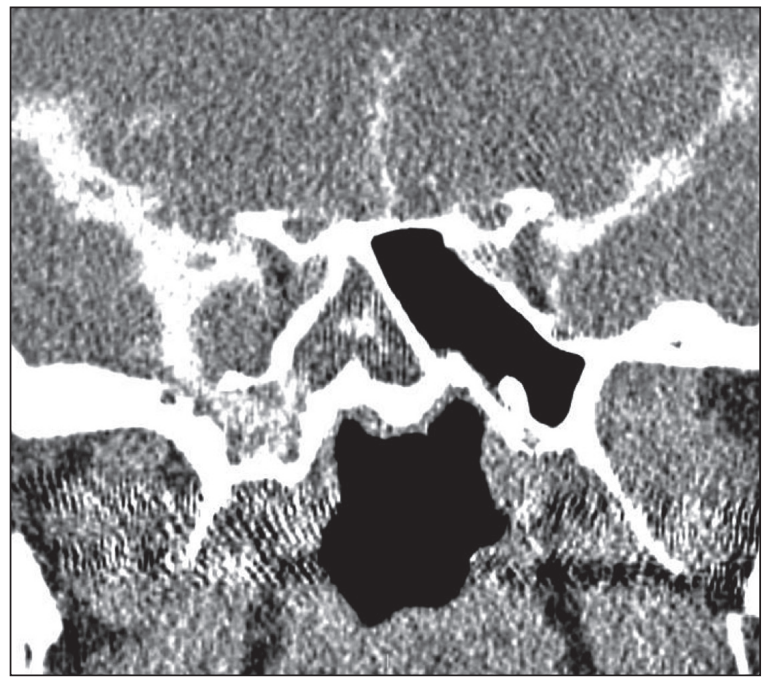

Figura3

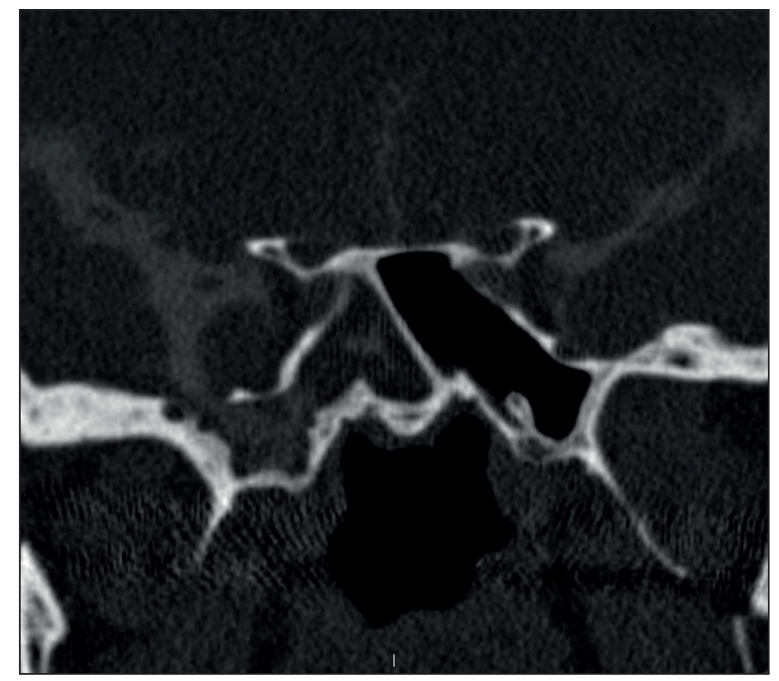

Figura 2.

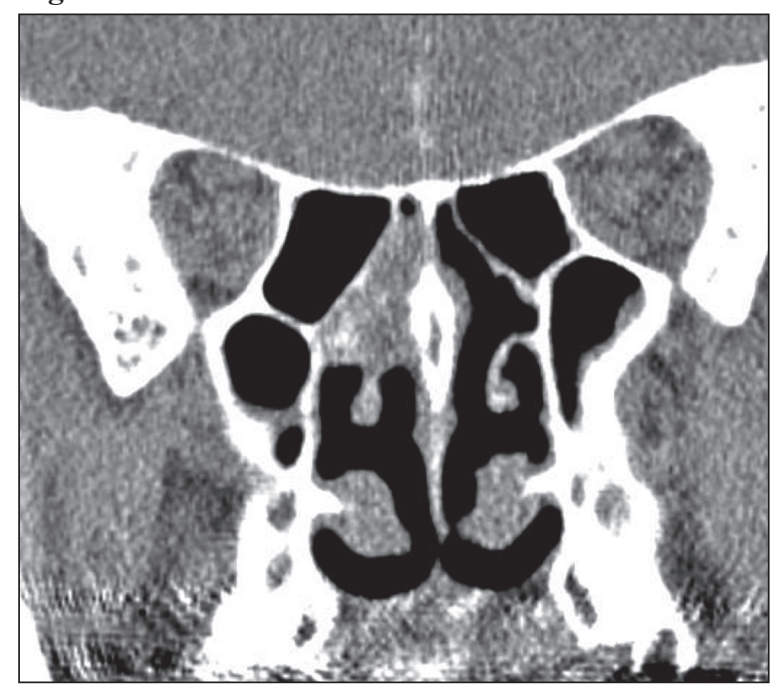

Figura 4.

\footnotetext{
1 Médico adjunto.

2 Cor. Médico.

Hospital Central de la Defensa Gómez-Ulla. Servicio de Radiodiagnóstico. Madrid. España.
}

Recibido: 24 de abril de 2012

Aceptado: 17 de julio de 2013 


\section{Diagnóstico: Fístula de líquido cefalorraquídeo (LCR) a través de un defecto óseo en la pared lateral derecha del seno esfenoidal}

En la cisternografía-TCMC se observa un gran desarrollo del seno esfenoidal que se extiende a la raíz de las alas mayores del esfenoides y de las apófisis pterigoides y una solución de continuidad de la pared lateral derecha del mismo (fig.2), a través de la cual se demuestra paso del medio de contraste hasta el seno esfenoidal (fig.3) y desde éste a las fosas nasales (fig.4). Estos hallazgos confirman la existencia de un defecto de la pared del mismo, con fuga de LCR.

Los defectos óseos se deben con mayor frecuencia a traumatismos de la base del cráneo; las fracturas del complejo frontoetmoidal y de la fosa craneal media son las que con más frecuencia se asocian a fugas de $\mathrm{LCR}^{1}$. También pueden ser debidas a procedimientos neuroquirúrgicos u otológicos como cirugía endoscópica de los senos paranasales o la resección transnasal de tumores hipofisarios². Entre las causas no traumáticas figuran los tumores que erosionan la base del cráneo, destacando los tumores hipofisarios como causa más frecuente de fuga no traumática ${ }^{1}$; otras posibles causas son la osteorradionecrosis inducida por radioterapia, procesos inflamatorios/infecciosos o lesiones congénitas que desarrollan hidrocefalia con fugas de LCR a través de zonas de debilidad de las estructuras óseas craneales.

Recientemente se describen las fugas espontáneas, sin factores de riesgo conocidos, que parecen ser más frecuentes en mujeres obesas de mediana edad y es frecuente que asocien Síndrome de la SillaTurca vacía ${ }^{2}$. En el caso presentado, el paciente no refiere antecedentes traumáticos y en el TC que se realizó inicialmente no se detectaron otras posibles causas, concluyendo que se trata de una fuga de LCR espontánea.

Las fugas espontáneas de LCR se producen en cualquier lugar de la base del cráneo, más frecuentemente en el techo del etmoides-lámina cribosa y en el seno esfenoidal ${ }^{2}$ con herniación de la dura madre y fuga de LCR a través de las zonas de debilidad anatómica. El diagnóstico preciso de la localización de una fuga de LCR es indispensable antes de planificar un tratamiento correcto $^{1-3}$. Además, las fugas de LCR a través del seno esfenoidal pueden ser difíciles de visualizar por endoscopia si se localizan en el receso lateral extremo que puede requerir un abordaje transcraneal ${ }^{4}$.

La TCMC es la prueba de imagen inicial ${ }^{1,3,5,6}$ para localizar los defectos óseos más pequeños permitiendo, tras una adquisición volumétrica, reconstrucciones multiplanares submilimétricas de la región de interés maxilofacial o temporal.

La cisternografía-TC se realiza actualmente con TCMC antes y después de inyectar contraste intratecal mediante punción lumbar. El paciente es colocado en Trendelemburg durante 90 minutos y se comprueba el paso del contraste intratecal a las cisternas basales por TC. En caso de fístula inactiva en el momento de la exploración, se puede evidenciar la fuga de LCR mediante maniobras de provocación (estornudo, posicionar la cabeza en prono inclinada) previos a la cisternografía-TCMC ${ }^{3}$. El resultado es positivo si se identifica el defecto óseo en la base del cráneo y la acumulación del contraste dentro del seno/fosa nasal u oído medio.

La RM cerebral no permite ver con seguridad los defectos óseos pequeños y suele reservarse para evaluar la posible existencia de un meningocele y/o meningoencefalocele a través del defecto óseo, difíciles de diferenciar de las secreciones intrasinusales en el estudio $\mathrm{TCMC}^{3}$.

\section{CONCLUSION}

Ante la sospecha clínica de fuga de LCR, las pruebas de imagen son esenciales para confirmar el diagnóstico de fuga, localizar con precisión el defecto óseo, estudiar sus posibles causas y planificar el tratamiento. La TCMC que incluya la base del cráneo y mastoides permite localizar el defecto óseo, confirmar el diagnóstico y suele ser suficiente cuando el defecto óseo es único. La Cisternografía-TC, con maniobras de provocación, si es necesario, está indicada actualmente en pacientes con múltiples defectos/fracturas base del craneo, con sospecha clínica de fuga de LCR, pero con TCMC inicial negativo y en pacientes con diagnóstico de fístula de LCR dudoso.

La RM cerebral debería realizarse en pacientes en los que la TCMC demostró un defecto óseo con posible meningocele o meningoencefalocele y es prioritaria en las fugas de LCR no traumáticas para su estudio etiológico (origen tumoral, infeccioso, etc).

\section{BIBLIOGRAFÍA}

1. Laine FJ, Nadel L, Braun IF. CT and MR Imaging of the central skull base. Part 2: Pathologic Spectrum. Radiographics. 1990 Sep;10(5):797-821.

2. Lloyd KM, DelGaudio JM, Hudgins PA. Imaging of Skull Base Cerebrospinal Fluid Leaks in Adults. Radiology. 2008 Sep;248(3):725-36.

3. Shetty PG, Shroff MM, Fatterpekar GM, Sahani DV, Kirtane MV. Retrospective Analysis of Spontaneous Sphenoid Sinus Fistula: MR and CT Findings. AJNR Am J Neuroradiol. 2000 Feb;21(2):337-42.

4. Anónimo. Fistula_de_liquido_cefalorraquideo. Revista de Neurocirugía Contemporánea. Disponible en: http://www.wikineurocirugia.com/doku. php?id=fistula_de_liquido_cefalorraquideo.

5. Stone JA, Castillo M, Neelon B, Mukherji SK. Evaluation of CSF Leaks: High-Resolution CT compared with Contrast-Enhanced CT and Radionuclide Cisternography. AJNR Am J Neuroradiol. 1999 Apr;20(4):706-12.

6. Zayas JO, Feliciano YZ, Hadley CR, Gomez AA, Vidal JA. Temporal bone trauma and the role of multidetector CT in the emergency department. Radiographics. 2011 Oct;31(6):1741-55. 